\title{
Hydrodynamic Pressure and Velocity Distributions in the Interlayer Crack of Ballastless Track under High-Speed Train Load: A Theoretical Analysis
}

\author{
Shihao Cao $\mathbb{D}^{1},{ }^{1}$ Shufang Zhai, ${ }^{1}$ Feng Dai, ${ }^{2}$ Shijie Deng, ${ }^{3}$ and Hui Wang ${ }^{1}$ \\ ${ }^{1}$ College of Civil Engineering and Architecture, Henan University of Technology, Zhengzhou 450001, China \\ ${ }^{2}$ China Railway Eryuan Engineering Group Co. Ltd., Chengdu 610031, China \\ ${ }^{3}$ MOE Key Laboratory of High-Speed Railway Engineering, Southwest Jiaotong University, Chengdu 610031, China \\ Correspondence should be addressed to Shihao Cao; 531148108@qq.com
}

Received 7 June 2018; Revised 30 July 2018; Accepted 8 August 2018; Published 19 August 2018

Academic Editor: Roberto G. Citarella

Copyright (C) 2018 Shihao Cao et al. This is an open access article distributed under the Creative Commons Attribution License, which permits unrestricted use, distribution, and reproduction in any medium, provided the original work is properly cited.

\begin{abstract}
In the abundant rain or poor drainage areas, there will be serious water damage existing in the interlayer of ballastless track. The essence of water damage of ballastless track is a dynamic development process of damage shape under the combined action of train load, hydrodynamic pressure, and flow velocity. In view of the distribution characteristics of hydrodynamic pressure and flow velocity in the interlayer crack of ballastless track under the high-speed train load, the simplified mechanics model for water and composite slab with interfacial crack is proposed in accordance with the water damage characteristics of ballastless track. Based on the conservation of mass and momentum theorem, the analytical expressions of water pressure and velocity in the saturated water crack were deduced. Similarly, the analytical expressions of water pressure and velocity in the unsaturated water crack were deduced by adding the state equation of ideal air. Considering that the water does not flow back timely, the analytical expressions of water pressure and velocity in another kind of unsaturated water crack were deduced. The results show that the hydrodynamic pressure and flow velocity in the interlayer crack are synthetically determined by multiple influencing factors such as fluid viscosity, load characteristic, crack shape, absolute pressure at the crack mouth, and initial volume of the air in the crack, and there is an intersecting phenomenon between influencing factors. When there is a small amount of air at the crack tip, the pressure and velocity distribution in the crack can be divided into three parts in terms of air-water interface and stagnation point. When the crack is filled with water, the hydrodynamic pressure tends to decrease along the direction of the crack mouth, and the distribution along the whole crack approximates to a cubic polynomial curve. Similarly, the flow velocity increases along the direction of crack mouth, and the distribution along the whole crack approximates to a quadratic polynomial curve.
\end{abstract}

\section{Introduction}

Ballastless track has become the main track structure of rapid transit railway because of its advantages of high comfort, high stability, and less maintenance. It has been developed rapidly and used widely. The design life of ballastless track is six decades which is estimated on the basis of the force characteristics acting on the track and the number of loads [1, 2]. However, the ballastless track, as the driving foundation, is exposed in the complex natural environment and bears the repeated action of train load, temperature load, rain erosion, and so on. These influencing factors make the component materials of the ballastless track in a continuous deterioration process, and the damage of ballastless track is inevitable [35]. The field investigation found that the damage rate in abundant rain or poor drainage areas is much faster than in dry areas; that is, the existence of water obviously reduces the life of the track.

Due to the differences of structural types, environmental factors, and operational conditions of ballastless track in various countries, their damage forms and related research priorities are also different [6]. The Japanese slab track adopts unit structure and takes the train load as the main design load without considering the influence of temperature. During 
the long-term operation, the track is in good condition, and the main damage forms are resin and CA mortar damage. Through analyzing the formation mechanism of damage, it is found that the longitudinal and transverse deformation of the track slab caused by the train load and the temperature load is the main cause of damage $[7,8]$. But the damage mechanism of the other ballastless track components has not been deeply studied.

The ballastless track in Germany is a longitudinal continuous structure. Temperature load and concrete shrinkage are the main considerations. The longitudinal reinforcement of the continuous concrete track bed is set in consideration of the temperature variation and the concrete shrinkage [911]. Where there is a possibility of interlayer separation, the pins are added to ensure enough interlayer bonding. The construction quality is strictly controlled under construction. Furthermore, the cracks are blocked timely and attention is paid to the drainage design. So far, the research on the water damage of ballastless track in Germany has not been reported.

In the early years, the integrated ballastless track in China was mainly used in tunnels, and the water damage of ballastless track caused by poor drainage of tunnels has ever happened [12]. Since the first comprehensive test section of ballastless track was built on Suining-Chongqing line in 2006, the ballastless track has developed rapidly and widely applied [13]. Because of the vast territory and large differences in geological and environmental conditions in China, there are many diseases on operational lines. These diseases include obvious damage caused by foundation deformation or extreme climate and also the water damage. Attention has been paid to the obvious damage caused by foundation deformation and extreme climate by the maintenance department and the researchers because of its clear cause and severe harm. A large number of related studies have been carried out at present [14-17]. The disease of ballastless track caused by water is a gradual process, and the initial damage will not cause serious consequences. Hence, not enough attention has been paid to this problem by the management department, and the related research has just started.

Aiming at the water damage of ballastless track, the CRTS series ballastless tracks were investigated nationally. On the basis of the field investigation data, the apparent characteristics and main causes of the water damage were clearly defined. The simplified mechanics model for water and composite slab with interfacial crack was presented according to the water damage characteristics, and the analytical expressions of hydrodynamic pressure and flow velocity in interlayer crack under the dynamic load were derived. The research results can provide hydrodynamic pressure and flow velocity boundary conditions for the further research on the dynamic evolution process of water damage shape of ballastless track.

\section{Characteristics and Control Factors of Water Damage of Ballastless Track}

The drainage problem is considered at the design stage of track structure. In general, when the rain falls onto the surface of ballastless track, most of water will drain through drainage facilities, and the remaining small amount of water will not affect the track structure. Through the nationwide investigation of the service situation of ballastless track, it is found that cracks are unavoidable on the surface or layer of the ballastless track due to the improper construction and maintenance and the long-term effect of train load and environmental factors, as shown in Figures 1(a) and 1(b). In the areas where the rain is rich or the drainage is poor, the water will seep into the cracks and become the precondition for the formation and development of the water diseases. Under the long-term soaking, the material mechanics properties of ballastless track will gradually decrease. A certain hydrodynamic pressure and flow velocity will be generated in the crack under the action of train load. Then the hydrodynamic pressure reacts on the crack surface and accelerates the crack propagation. Meanwhile, the flow velocity acting on the surface of the cracks will scour the surface particles repeatedly. The surface particles are exfoliated and result in a gradual increase of crack opening and the oozing slurry phenomenon at the crack mouth, as shown in Figures 1(c) and 1(d). Under the combined action of material softening, crack tip expansion, and crack surface scouring, the water damage of ballastless track will be further developed into more obvious diseases, such as sleeper loosening, void under track slab, or track bed, as shown in Figures 1(e) and 1(f).

\section{Simplified Mechanical Model of Fluid-Structure Interaction}

Hydrodynamic pressure and velocity are the driving forces for the development of ballastless track water diseases, and clarifying their distribution characteristics is the precondition for the study of the development mechanism of water diseases. However, due to the complexity of fluid boundary conditions and the difficulty of numerical simulation, it is difficult to obtain the analytical expressions of three-dimensional hydrodynamic pressure and flow velocity distribution in the crack. In addition, the hydrodynamic pressure and flow velocity in the crack of ballastless track are synthetically determined by multiple factors, and the factors will have cross effects on each other [18], which means that the formation and variation of velocity are too complex to be analyzed with complete numeric models. Therefore, the complete numeric model usually needs to be simplified in order to analyze conveniently.

Through the numerical analysis, it is found that the flow velocity distribution in the interlaminar crack of ballastless track is a three-dimensional problem, but the longitudinal and vertical components of flow velocity are significantly less than lateral component of flow velocity, which means that the effect of longitudinal and vertical components on velocity distribution is very limited. Therefore, it is also feasible to simplify the mechanical model into two dimensions when the small longitudinal flow of water (out-of-plane velocity) is ignored in this paper, as shown in Figure 2, where $L$ is the crack depth and $a$ is the crack opening. 


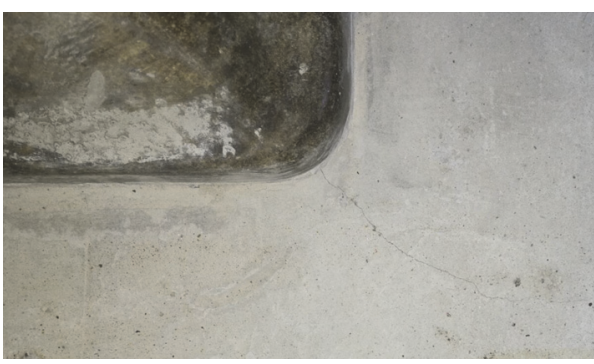

(a)

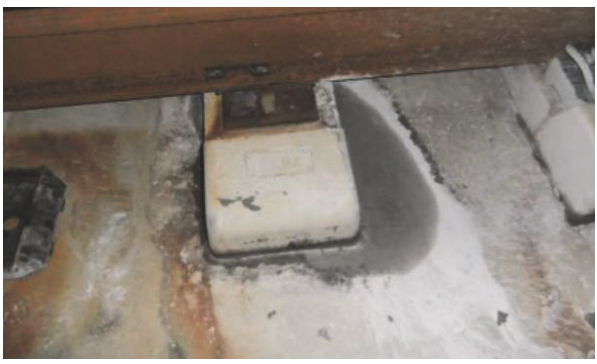

(c)

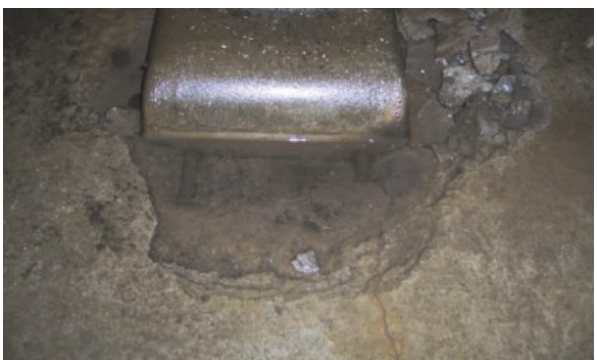

(e)

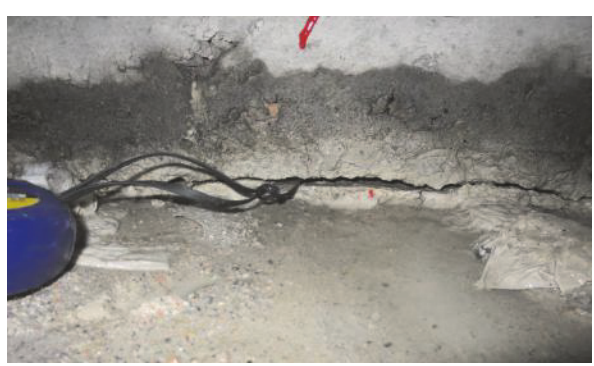

(b)

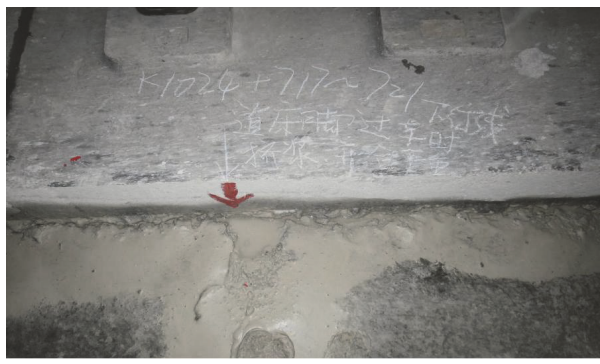

(d)

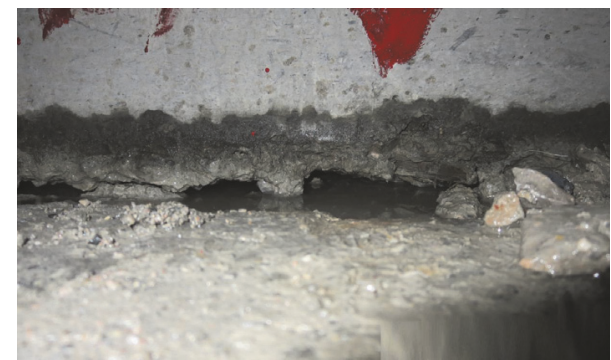

(f)

FIGURE 1: Development process of water damage: (a) surface crack; (b) interlayer crack; (c) oozing slurry at sleeper; (d) oozing slurry in interlayer crack; (e) sleeper loosening; (f) void under track bed.

In the model shown in Figure 2, the crack is assumed to be located at the interface of the composite slab. The crack is filled with water, and the compressibility of water is neglected. Without considering the influence of the elastic foundation, the fixed constraint is applied at the bottom of the model.

\section{Analytical Expression for Hydrodynamic Pressure and Velocity in Saturated Water Crack}

As a common liquid in nature, the movement of water under the compression of crack surfaces must obey the most basic and universal motion laws, such as the mass conservation and the momentum theorem. For the incompressible fluid, the integral expression of the mass conservation law is $[19,20]$

$$
\frac{d m}{d t}=\rho \frac{d}{d t} \int_{A} d A+\rho \oint_{\Gamma}(\mathbf{u} \cdot \mathbf{n}) d \Gamma=0
$$

The integral expression of the momentum theorem is

$$
\frac{d \mathbf{M}}{d t}=\rho \frac{d}{d t} \int_{A} \mathbf{u} d A+\rho \oint_{\Gamma} \mathbf{u}(\mathbf{u} \cdot \mathbf{n}) d \Gamma=\sum \mathbf{F}
$$

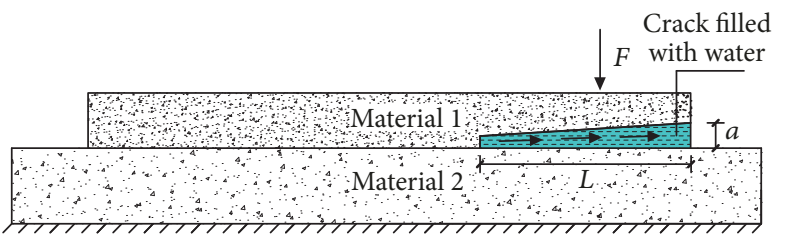

Figure 2: Simplified mechanical model of fluid-structure interaction.

where $m$ is the mass of liquid, $t$ is the time, $\rho$ is the density of liquid, $A$ is the area of the control volume, $\mathbf{u}$ is the velocity vector of liquid, $\mathbf{n}$ is the outer normal direction of the control volume boundary, $\Gamma$ is the control volume boundary, $\mathbf{M}$ is the momentum of liquid, and $\mathbf{F}$ is the force vector acting on the control volume.

The concrete strength grade of ballastless track slab can reach up to C55 and belongs to high strength concrete. It can be known from the uniaxial tensile test that [21] when the stress is less than 70 percent of concrete tensile strength, the constitutive relation is basically linear. Actually, the design 


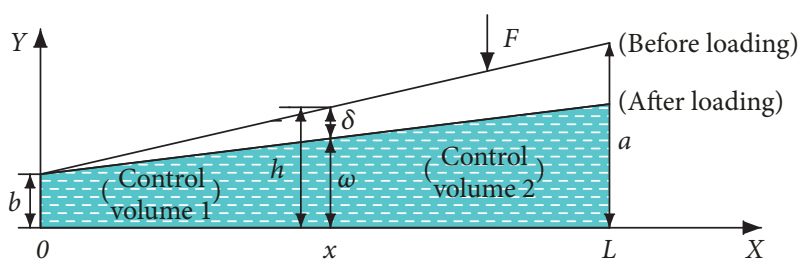

FIGURE 3: Schematic diagram of crack shape before and after loading.

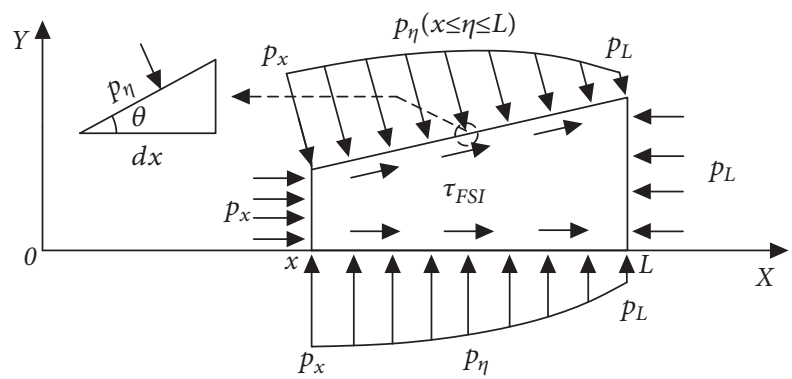

FIgURE 4: Schematic diagram of forces acting on control volume 2.

of ballastless concrete track slab adopts the allowable stress method, and its safety is too conservative [22]. Under the action of train load, the mechanical behavior of concrete track slab can still be approximately linear. In the elastic range, the upper surface displacement $\delta$ of the crack in Figure 3 is linearly related to the load $F$. That is,

$$
\delta(x)=F \cdot \beta(x)
$$

When the crack shape and load position are unchanged, $\beta(x)$ is a determined distribution function.

Before loading, the crack opening $h$ at the position $x$ is

$$
h(x)=b+\frac{(a-b) x}{L}
$$

After loading, the crack opening $\omega$ at the position $x$ is

$$
\omega(x)=b+\frac{a-b}{L} x+F \cdot \beta(x)
$$

When considering the effect of fluid viscosity, the flow state of water in the interlaminar crack of ballastless track can be determined by Reynolds number (Re). The flow state is laminar flow when the Reynolds number $\operatorname{Re}<2300$, and the flow state is turbulence flow when the Reynolds number Re $>$ 2300 . For the laminar flow, the velocity $v$ of arbitrary section in crack is parabolic [23]; thus

$$
v(x, y)=v_{m}(x)\left\{1-\frac{[2 y-\omega(x)]^{2}}{\omega^{2}(x)}\right\}
$$

The relation between the maximum flow velocity $v_{m}$ and the average velocity $\bar{v}$ of arbitrary section is as follows:

$$
\bar{v}(x)=\frac{2}{3} v_{m}(x)
$$

Select control volume 1 (fluid domain: $0 \sim x$ ) shown in Figure 3 , combined with the formulas (5), (6), and (7). Then,

$$
\begin{gathered}
\rho \frac{d}{d t} \int_{A} d A=\rho \frac{d}{d t} \int_{0}^{x} \omega(x) d x=\rho F^{\prime} \int_{0}^{x} \beta(x) d x \\
\rho \oint_{\Gamma}(\mathbf{u} \cdot \mathbf{n}) d \Gamma=\rho \int_{0}^{\omega(x)} v(x, y) d y=\frac{2}{3} \rho v_{m}(x) \omega(x)
\end{gathered}
$$

Substitute formulas (8) and (9) into formula (1), the following can be obtained:

$$
v_{m}(x)=-\frac{3 F^{\prime}}{2 \omega(x)} \int_{0}^{x} \beta(x) d x
$$

Equation (10) is the analytical expression of water velocity distribution in crack under the dynamic load. According to the analytical expression, the water velocity in the crack is not only affected by the geometric shape of the crack, but also sensitive to the first derivative of the loading function.

Control volume 2 shown in Figure 3 (fluid domain: $x \sim L$ ) is selected as an object of study, and the forces acting on the control volume are shown in Figure 4.

Here $\tau_{F S I}$ is the shear stress in the fluid-structure interaction surface; $p_{L}$ is the pressure at the crack mouth; $p_{x}$ is the water pressure at the abscissa of $x$.

For Newton fluid, the relationship between shear stress and velocity gradient is linear [23], as shown in the following formula:

$$
\tau(x, y)=\mu \frac{d v(x, y)}{d y}
$$

where $\mu$ is the dynamic viscosity coefficient

The shear stress $\tau_{F S I}$ along the boundary can be obtained by formulas (6) and (11):

$$
\tau_{F S I}(x)=\frac{4 \mu v_{m}(x)}{\omega(x)}
$$

The component of the shear force acting on control volume 2 along the $x$ direction is

$$
\begin{gathered}
\int_{x}^{L} \tau_{F S I}(x) d x+\int_{x}^{L} \tau_{F S I}(x) \frac{d x}{\cos (\theta)} \cos (\theta) \\
=12 \mu F^{\prime} \int_{x}^{L} \frac{d x}{\omega^{2}(x)} \int_{0}^{x} \beta(x) d x
\end{gathered}
$$

When $\delta<<h$, the angle between two crack faces is approximately

$$
\tan (\theta) \approx \frac{a-b}{L}
$$

According to Newton's third law, the normal pressure acting on the control volume of the solid boundary is equal to the fluid pressure $p$, and the component of the normal pressures acting on control volume 2 along the $x$ direction is

$$
\int_{x}^{L} p(x) \frac{d x}{\cos (\theta)} \sin (\theta)=\frac{a-b}{L} \int_{x}^{L} p(x) d x
$$


The component of the resultant force acting on control volume 2 along the $x$ direction is

$$
\begin{aligned}
\sum F_{x}= & p(x) \omega(x)+\frac{a-b}{L} \int_{x}^{L} p(x) d x-p_{L} \omega(L) \\
& -12 \mu F^{\prime} \int_{x}^{L} \frac{d x}{\omega^{2}(x)} \int_{0}^{x} \beta(x) d x
\end{aligned}
$$

It can be obtained according to formulas (5) and (6) that

$$
\begin{aligned}
\rho \frac{d}{d t} \int_{A} \mathbf{u} d A & =\rho \frac{d}{d t} \int_{x}^{L} \bar{v}(x) \omega(x) d x \\
\rho \oint_{\Gamma} \mathbf{u}(\mathbf{u} \cdot \mathbf{n}) d \Gamma & =\frac{6}{5} \rho\left[\bar{v}^{2}(L) \omega(L)-\bar{v}^{2}(x) \omega(x)\right]
\end{aligned}
$$

Substitute formulas (16), (17), and (18) into formula (2), and obtain

$$
\begin{aligned}
& \frac{a-b}{L} \int_{x}^{L} p(x) d x+p(x) \omega(x) \\
& =-\underbrace{\rho F^{\prime \prime} A(x)}_{A}+\underbrace{1.2 \rho\left(F^{\prime}\right)^{2} B(x)}_{B}+\underbrace{12 \mu F^{\prime} C(x)}_{C} \\
& \quad+\underbrace{p_{L} \omega(L)}_{D}
\end{aligned}
$$

where

$$
\begin{aligned}
& A(x)=\int_{x}^{L} d x \int_{0}^{x} \beta(x) d x \\
& B(x)=\frac{\left[\int_{0}^{L} \beta(x)\right]^{2}}{\omega(L)}-\frac{\left[\int_{0}^{x} \beta(x)\right]^{2}}{\omega(x)} \\
& C(x)=\int_{x}^{L} \frac{d x}{\omega^{2}(x)} \int_{0}^{x} \beta(x) d x
\end{aligned}
$$

Formula (19) is the analytical formula of the hydrodynamic pressure in interlayer crack of laminate under dynamic load. From the analytical expression, the hydrodynamic pressure not only is influenced by the geometric shape of the crack, the fluid viscosity, the fluid density, and the atmosphere, but also is sensitive to the first and second derivative of the loading function.

\section{Analytical Expression for Hydrodynamic Pressure and Velocity in Unsaturated Water Crack}

In fact, there may be a certain amount of air in the crack before loading; that is, the crack will not be full of water, which is called unsaturated water crack. Under the action of dynamic load, the flow and pressure distribution of fluids including water and air in the crack are shown in Figure 5.

Here $L$ is the crack depth, $L_{a p}$ is the air depth in the crack after loading, $L_{\text {sta }}$ is the distance from stagnation point to the crack tip, $p_{a i}$ is the initial pressure of air in the crack, $p_{a p}$ is

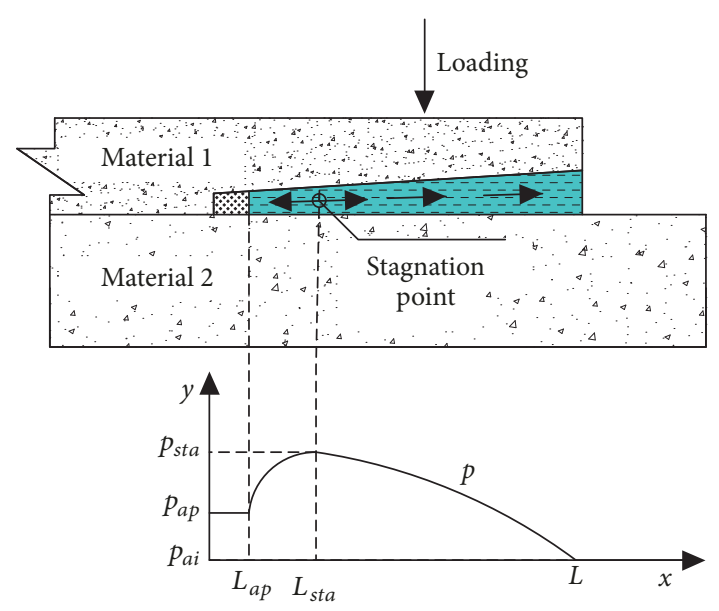

FIGURE 5: Flow condition and pressure distribution of fluids including water and air in the crack.

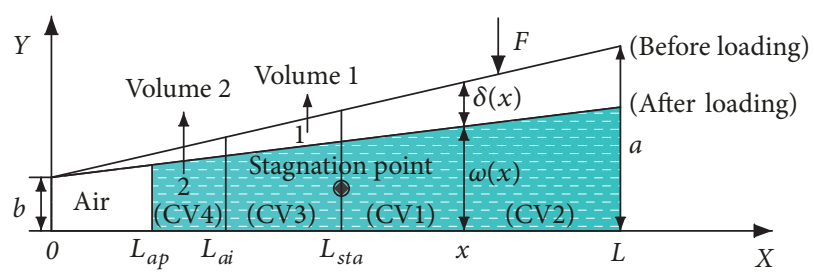

FIGURE 6: Schematic diagram of crack deformation.

the pressure at the interface between air and water, and $p_{s t a}$ is the water pressure at the stagnation point.

Under the action of dynamic load, the distribution of hydrodynamic pressure in saturated water crack is a typical two-phase coupling problem of liquid and solid, and the pressure distribution in the unsaturated water crack is a typical three-phase coupling problem of liquid, solid, and gas. Under the action of dynamic load, there will be a point with the flow velocity $v=0$ in the unsaturated water crack, which is called stagnation point, as shown in Figure 5. While loading, the liquid flows from stagnation point to two opposite directions and tends to increase along two opposite directions. The location of stagnation point is not fixed but will change with the initial volume of air in the crack. The shape change of the unsaturated water crack before and after loading is shown in Figure 6.

Here $a$ is the initial crack opening at the crack mouth, $\omega(x)$ is the crack opening at the position $x$ after loading, $\delta(x)$ is the upper surface displacement of the crack at the position $x$ after loading, $L_{a i}$ is the air depth in the unsaturated water crack at the initial time, and $F$ is the force acting on the solid structure.

5.1. Analytical Expression of Hydrodynamic Pressure and Velocity at the Stage $0 \sim L_{s t a}$. At the initial time, the interface between air and water in unsaturated water crack is at $x=L_{a i}$, 
and the initial volume $V_{a i}$ of the air field in crack can be obtained by

$$
V_{a i}=\frac{[2 b L-(a-b) x] L_{a i}}{2 L}
$$

Before loading, the initial pressure in the two regions (air and water) is equal to the atmospheric pressure $p_{L}$ because the atmosphere, water domain, and air domain exiting in the crack are in the static equilibrium state.

After loading, the interface between air and water moves from $L_{a i}$ to $L_{a p}$. On the basis of the incompressibility of water, it is known that volume 1 is equal to volume 2; that is,

$$
\int_{L_{a p}}^{L_{a i}} \omega(x) d x=\int_{L_{a i}}^{L_{s t a}} \delta(x) d x
$$

The volume change of the air region, $\Delta V$, is as follows:

$$
\Delta V=\int_{0}^{L_{\text {sta }}} \delta(x) d x=F \int_{0}^{L_{\text {sta }}} \beta(x) d x
$$

According to the state equation of ideal gas, the product of the pressure and volume of the gas is a constant when the mass and temperature of the gas remain unchanged [23]; that is,

$$
p V=C
$$

Combined with formulas (21), (23), and (24), the pressure $p_{a i r}$ of the air region under dynamic load is

$$
\begin{aligned}
p_{a i r} & =\frac{p_{a i} \cdot V_{a i}}{V_{a i}+\Delta V} \\
& =\frac{p_{a i} \cdot[2 b L+(a-b) x] L_{a i}}{[2 b L+(a-b) x] L_{a i}+2 L F \int_{0}^{L_{s t a}} \beta(x) d x}
\end{aligned}
$$

The pressure $p_{a p}$ at the interface between air and water is equal to the pressure in the air region.

$$
p_{a p}=p_{a i r}
$$

5.2. Analytical Expression of Hydrodynamic Pressure and Velocity at the Stage $L_{\text {sta }} \sim L$. Select the control volume 1 shown in Figure 6 (fluid domain: $L_{\text {sta }} \sim x$ ), combined with formula (1), (5), (6) and (7). The following can be obtained:

$$
v_{m}(x)=-\frac{3 F^{\prime}}{2 \omega(x)} \int_{L_{s t a}}^{x} \beta(x) d x
$$

Formula (27) is the analytical expression of flow velocity at the stage $L_{\text {sta }} \sim L$ in unsaturated water crack under the dynamic load.

Selecting the control volume 2 shown in Figure 6 (fluid domain: $x \sim L$ ) as a research object, the force diagram is shown in Figure 4. The component of resultant force acting on the control volume along the $x$ direction is

$$
\begin{aligned}
\sum F_{x}= & p(x) \omega(x)+\frac{a-b}{L} \int_{x}^{L} p(x) d x-p_{L} \omega(L) \\
& -12 \mu F^{\prime} \int_{x}^{L} \frac{d x}{\omega^{2}(x)} \int_{L_{s t a}}^{x} \beta(x) d x
\end{aligned}
$$

Substitute formulas (5), (27), and (28) into (2), and obtain

$$
\begin{aligned}
& \frac{a-b}{L} \int_{x}^{L} p(x) d x+p(x) \omega(x) \\
& =-\underbrace{\rho F^{\prime \prime} A_{1}(x)}_{A}+\underbrace{1.2 \rho\left(F^{\prime}\right)^{2} B_{1}(x)}_{B}+\underbrace{12 \mu F^{\prime} C_{1}(x)}_{C} \\
& \quad+\underbrace{p_{L} \omega(L)}_{D}
\end{aligned}
$$

where $A_{1}(x)=\int_{x}^{L} d x \int_{L_{s t a}}^{x} \beta(x) d x$.

$$
\begin{aligned}
& B_{1}(x)=\frac{\left[\int_{L_{s t a}}^{L} \beta(x)\right]^{2}}{\omega(L)}-\frac{\left[\int_{L_{s t a}}^{x} \beta(x)\right]^{2}}{\omega(x)} \\
& C_{1}(x)=\int_{x}^{L} \frac{d x}{\omega^{2}(x)} \int_{L_{s t a}}^{x} \beta(x) d x
\end{aligned}
$$

Formula (29) is the analytical expression of hydrodynamic pressure at the stage $L_{\text {sta }} \sim L$ in unsaturated water crack under dynamic load.

5.3. Analytical Expression of hydrodynamic Pressure and Velocity at the Stage $L_{a p} \sim L_{\text {sta }}$. Similarly, control volume 3 shown in Figure 6 was selected to obtain the analytical expression of flow velocity at the stage $L_{a p} \sim L_{s t a}$ :

$$
v_{m}(x)=-\frac{3 F^{\prime}}{2 \omega(x)} \int_{x}^{L_{s t a}} \beta(x) d x
$$

Selecting control volume 4, the analytical expression of hydrodynamic pressure at this stage can be obtained as follows:

$$
\begin{aligned}
& \frac{a-b}{L} \int_{x}^{L} p(x) d x-p(x) \omega(x) \\
& =-\underbrace{\rho F^{\prime \prime} A_{2}(x)}_{A}+\underbrace{1.2 \rho\left(F^{\prime}\right)^{2} B_{2}(x)}_{B}-\underbrace{12 \mu F^{\prime} C_{2}(x)}_{C} \\
& \quad-\underbrace{p_{a p} \omega\left(L_{a p}\right)}_{D}
\end{aligned}
$$

where

$$
\begin{aligned}
& A_{2}(x)=\int_{L_{a p}}^{x} d x \int_{x}^{L_{s t a}} \beta(x) d x \\
& B_{2}(x)=\frac{\left[\int_{L_{a p}}^{L_{\text {sta }}} \beta(x)\right]^{2}}{\omega\left(L_{a p}\right)}-\frac{\left[\int_{x}^{L_{s t a}} \beta(x)\right]^{2}}{\omega(x)} \\
& C_{2}(x)=\int_{L_{a p}}^{x} \frac{d x}{\omega^{2}(x)} \int_{x}^{L_{s t a}} \beta(x) d x
\end{aligned}
$$

Formula (32) is the analytical expression of hydrodynamic pressure at stage $L_{a p} \sim L_{s t a}$ in the unsaturated water crack under dynamic load. 


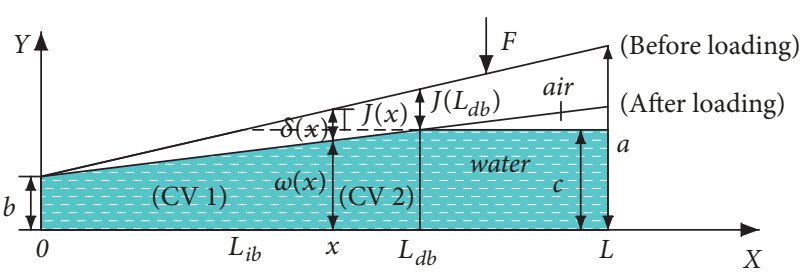

FIGURE 7: Schematic diagram of crack deformation.

According to formulas (27) and (31), the flow velocity in unsaturated water crack is influenced by not only the crack geometry and load characteristics, but also the position of the stagnation point in the crack. The stagnation point is mainly determined by the initial volume of the air inside the crack. In terms of the formulas (29) and (32), the hydrodynamic pressure in unsaturated water crack is influenced by not only the geometric shape of the crack, the fluid viscosity, the fluid density, and the absolute pressure at crack mouth, but also the position of the stagnation point in the crack.

When the initial volume of the air region in the crack is determined, the flow velocity distribution in the crack can be obtained by formulas (6), (27), and (31). Similarly, the hydrodynamic pressure distribution in the whole crack can be obtained by formulas (25), (29), and (32).

\section{Analytical Expression for Hydrodynamic Pressure and Velocity in Another Kind of Unsaturated Water Crack}

For the saturated water crack as shown in Figure 2, the water will periodically outflow and flow back due to the extrusion and pumping action of crack surfaces, respectively. When the water can not flow back timely, there is going to be another kind of unsaturated water crack, as shown in Figure 7.

Here $c$ is the liquid level, $J(x)$ is the distance between liquid level and crack upper surface at the position $x, L_{i b}$ is the position of intersection point between liquid level and crack upper surface before loading, and $L_{d b}$ is the position of intersection point between liquid level and crack upper surface after loading. In order to distinguish from the crack mouth $L, L_{d b}$ is defined as fluid outlet in this paper.

According to the crack geometry and liquid boundary parameter in the crack, $J(x)$ can be obtained:

$$
J(x)=h(x)-c=\omega(x)+\delta(x)-c
$$

In fact, the position of $L_{d b}$ is not fixed, but moving under the dynamic load. The position should satisfy

$$
J\left(L_{d b}\right)=\delta\left(L_{d b}\right)
$$

Combined with formulas (3), (4), (34), and (35), the following is obtained:

$$
\frac{(a-b) L_{d b}}{L}+b-c=F \beta\left(L_{d b}\right)
$$

In this case, the hydrodynamic pressure and flow velocity distributions in the crack can be divided into two regions in terms of fluid outlet $L_{d b}: 0 \sim L_{d b}$ and $L_{d b} \sim L$. For the water in region $L_{d b} \sim L$, the value of hydrodynamic pressure is approximately equal to the atmospheric pressure due to the direct contact with the atmosphere.

For the water in region $0 \sim L_{d b}$, the hydrodynamic pressure and flow velocity distribution expressions can be derived on the basis of the same method as saturated water crack, as shown in Section 4 in this paper. Selecting the control volume 1 shown in Figure 7 (fluid domain: $0 \sim x$ ), the analytical expression of flow velocity can be obtained as follows:

$$
v_{m}(x)=-\frac{3 F^{\prime}}{2 \omega(x)} \int_{0}^{x} \beta(x) d x
$$

Similarly, selecting control volume 2 (fluid domain: $x$ $L_{d b}$ ), the analytical expression of hydrodynamic pressure can be obtained as follows:

$$
\begin{aligned}
& \frac{a-b}{L} \int_{x}^{L_{d b}} p(x) d x+p(x) \omega(x) \\
& =-\underbrace{\rho F^{\prime \prime} A_{3}(x)}_{A}+\underbrace{1.2 \rho\left(F^{\prime}\right)^{2} B_{3}(x)}_{B}+\underbrace{12 \mu F^{\prime} C_{3}(x)}_{C} \\
& \quad+\underbrace{p\left(L_{d b}\right) \omega\left(L_{d b}\right)}_{D}
\end{aligned}
$$

where

$$
\begin{aligned}
& A_{3}(x)=\int_{x}^{L_{d b}} d x \int_{0}^{x} \beta(x) d x \\
& B_{3}(x)=\frac{\left[\int_{0}^{L_{d b}} \beta(x)\right]^{2}}{\omega\left(L_{d b}\right)}-\frac{\left[\int_{0}^{x} \beta(x)\right]^{2}}{\omega(x)} \\
& C_{3}(x)=\int_{x}^{L_{d b}} \frac{d x}{\omega^{2}(x)} \int_{0}^{x} \beta(x) d x
\end{aligned}
$$

\section{Simplification of Analytical Expression}

When the crack is filled with water and $b=a$ (the crack shape is rectangular), the analytical expression (38) of the hydrodynamic pressure can be transformed into formula (41) under the sinusoidal load shown in the following formula:

$$
F=F_{m}+F_{o} \sin (2 \pi f t)
$$

where $F_{m}$ is the average load, $F_{o}$ is the loading amplitude, and $f$ is the loading frequency.

$$
\begin{aligned}
p(x)= & \underbrace{\left(4 \pi^{2} \rho f^{2} F_{o} \sin (2 \pi f t) / a\right) A_{4}(x)}_{A} \\
& +\underbrace{\left(24 \pi^{2} \rho f^{2} F_{o}{ }^{2} \cos ^{2}(2 \pi f t) / 5 a^{2}\right) B_{4}(x)}_{B} \\
& +\underbrace{\left(24 \pi \mu f F_{o} \cos (2 \pi f t) / a^{3}\right) A_{4}(x)}_{C}+p_{L}
\end{aligned}
$$


where

$$
\begin{aligned}
& A_{4}(x)=\int_{x}^{L} d x \int_{0}^{x} \beta(x) d x \\
& B_{4}(x)=\left[\int_{0}^{L} \beta(x)\right]^{2}-\left[\int_{0}^{x} \beta(x)\right]^{2}
\end{aligned}
$$

The item $A$ of formula (41) is produced by the mutual extrusion of the fluid. The term $B$ is produced by the shape change of the crack relative to the initial shape, and the term $C$ is caused by the fluid viscosity. When $\delta<<h$, the hydrodynamic pressure generated by the change of the crack shape is very small. Therefore, the effect of the term $B$ on the hydrodynamic pressure can be negligible.

In order to further study the primary and secondary relationship between terms $A$ and $C$ in formula (41), the ratio $R$ of the maximum value of term $C$ to the maximum value of term $A$ is supposed; that is,

$$
R=\frac{6 \mu}{\pi \rho a^{2} f}
$$

In view of formula (43), it is known that when the fluid properties (fluid viscosity $\mu$ and density $\rho$ ) are certain, $R$ is affected by the loading frequency $f$ and the crack opening $a$ together. With the increase of the loading frequency and the crack opening, the influence of viscosity decreases gradually. Through analysis of formula (43), it is found that the influence of the water viscosity on the pressure is negligible basically when the crack opening $a>2 \mathrm{~mm}$ under the high frequency dynamic load. In this case, the hydrodynamic pressure is mainly determined by term $A$ of analytical expression (41), and formula (41) can be simplified to the following formula:

$$
p(x)=\frac{4 \pi^{2} \rho f^{2} F_{o} \sin (2 \pi f t)}{a} A(x)+p_{L}
$$

Formula (44) indicates that the pressure $p$ in the interlayer crack is linear with the loading amplitude $F_{o}$ under the dynamic load and has a square relationship with the loading frequency $f$ and is inversely proportional to the crack opening $a$.

Similarly, substitute formula (40) into formula (37). The following can be obtained:

$$
v_{m}(x)=-\frac{3 \pi f F_{o} \cos (2 \pi f t)}{a} D(x)
$$

where $D(x)=\int_{0}^{x} \beta(x) d x$.

Formula (45) shows that the flow velocity $v$ in the crack is linear with the loading amplitude $F_{o}$ under the dynamic load and has a linear relationship with the loading frequency $f$ and is inversely proportional to the crack opening $a$.

\section{A Numerical Example}

In order to verify the correctness of the analytical formula of hydrodynamic pressure, the model shown in Figure 8

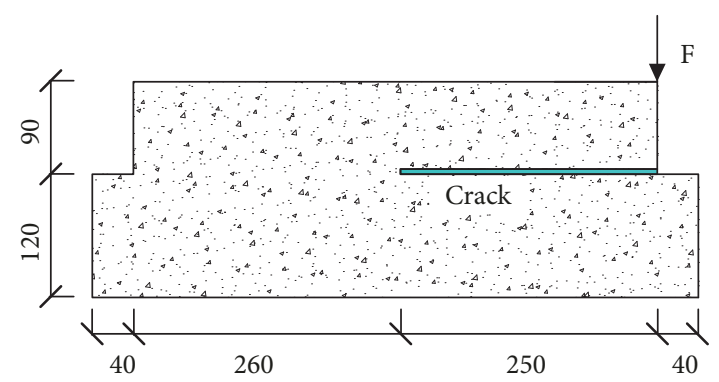

FIgURE 8: Calculation model of hydrodynamic pressure in the interlayer crack of concrete $(\mathrm{mm})$.

was taken as a numerical example. The calculation model of hydrodynamic pressure is composed of two parts: solid domain (concrete specimen with interlayer crack) and fluid domain (water in crack), and its corresponding ANSYS-CFX model is shown in Figure 9. The mesh size of the solid domain far from the crack is $10 \mathrm{~mm}$, while near the crack it is $0.25 \mathrm{~mm}$. The mesh size of the fluid domain along the depth direction is $0.25 \mathrm{~mm}$, and that at the vertical opening direction is less than $0.2 \mathrm{~mm}$.

The solid material of calculation model is C30 concrete, and its elasticity modulus and Poisson's ratio are $30 \mathrm{GPa}$ and 0.2 , respectively. The fluid in the crack is water, and its density $\rho=998.2 \mathrm{~kg} / \mathrm{m}^{3}$; the dynamic viscosity coefficient $\mu=$ $1.002 \times 10^{-3}$ pa.s. The reference pressure at the crack mouth is $0 \mathrm{~Pa}$. The peak value of the hydrodynamic pressure at different positions was obtained by formula (41) and ANSYSCFX under the sinusoidal load with loading frequency $f=10$ $\mathrm{Hz}$ and loading amplitude $F_{o}=1 \mathrm{kN}$, as shown in Table 1 , where $\mathrm{x}$ represents the distance between the calculated point and the crack tip.

As shown in Table 1, the peak value of hydrodynamic pressure calculated by the item $A$ is much larger than those calculated by items $B$ and $C$ of hydrodynamic pressure analytical expression (41). This phenomenon indicates that the hydrodynamic pressure in the crack is mainly determined by item $A$ of equation (41) under the dynamic load. The primary and secondary influence relation of all the items is consistent with the theoretical analysis results. As can be seen from Figure 10(a), the water pressure in the interlayer crack of concrete decreases along the direction of crack mouth, and the peak value appears at the crack tip. The distribution law can be explained based on the conservation of mass and momentum theorem. When the water is pressed by the crack surfaces, it is known from the mass conservation of the fluid that the flow velocity along the crack mouth will increase gradually, as shown in Figure 10(b). The increase of flow velocity also represents the increase of momentum. According to the momentum theorem, the direction of the resultant force acting on the control volume is in accordance with the direction of momentum increase. This is the reason that water pressure will decrease along the direction of crack mouth. In addition, the peak value of hydrodynamic pressure calculated by analytical expression is $67.37 \mathrm{~Pa}$, and the numerical value is $62.56 \mathrm{~Pa}$. The similarity between analytical 
TABLE 1: Comparison of analytical and numerical results.

\begin{tabular}{|c|c|c|c|c|c|}
\hline \multirow{2}{*}{$\mathrm{x} / \mathrm{m}$} & \multicolumn{4}{|c|}{ analytical results } & \multirow{2}{*}{$\begin{array}{c}\text { numerical results } \\
\mathrm{P}_{\max } / \mathrm{Pa}\end{array}$} \\
\hline & $\mathrm{A}_{\max } / \mathrm{Pa}$ & $\mathrm{B}_{\max } / \mathrm{Pa}$ & $\mathrm{C}_{\max } / \mathrm{Pa}$ & $\mathrm{P}_{\max } / \mathrm{Pa}$ & \\
\hline 0 & 67.37 & 0.2154 & 1.435 & 67.37 & 62.56 \\
\hline 0.05 & 65.75 & 0.215 & 1.401 & 65.75 & 61.82 \\
\hline 0.1 & 61.55 & 0.2106 & 1.311 & 61.55 & 58.55 \\
\hline 0.15 & 51.69 & 0.1912 & 1.101 & 51.69 & 49.61 \\
\hline 0.2 & 32.57 & 0.134 & 0.694 & 32.57 & 31.53 \\
\hline 0.25 & 0 & 0 & 0 & 0 & 0.001 \\
\hline
\end{tabular}

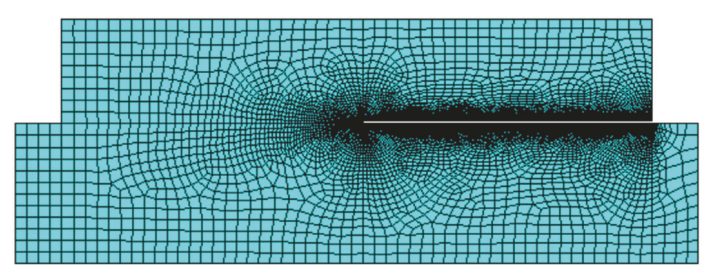

(a)

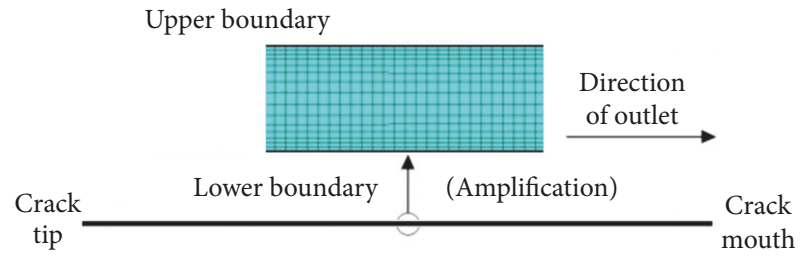

(b)

FIGURE 9: Finite calculation model: (a) solid domain; (b) fluid domain.

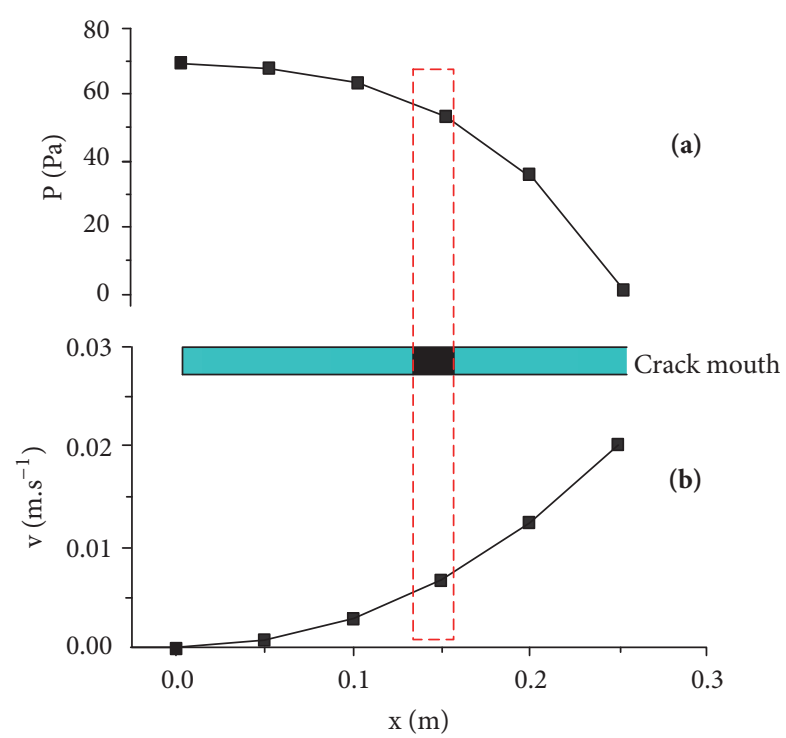

FIGURE 10: Distribution of water pressure and velocity along the direction of crack mouth: (a) pressure; (b) velocity.

and numerical results can reach $92.86 \%$. The consistency between analytical and numerical simulation results shows that the analytical formulas of hydrodynamic pressure were correctly derived.

\section{Conclusions}

In this paper, the simplified mechanics model for water and composite slab with interfacial crack was proposed in accordance with the water damage characteristics of ballastless track. Based on the conservation of mass and momentum theorem, the analytical expressions of water pressure and velocity in the saturated water crack were deduced. Similarly, the analytical expressions of water pressure and velocity in the unsaturated water crack were deduced by adding the state equation of ideal air. Considering the water not flowing back timely, the analytical expressions of water pressure and velocity in another kind of unsaturated water crack were deduced. In order to verify the correctness of the theoretical derivation, the ANSYS Workbench was used to conduct fluidstructure interaction analysis, and the similarity between analytical and numerical results can reach $92.86 \%$. According to the theoretical results, the primary and secondary influence relationship between multiple factors and the mechanism of intersecting action are determined. The research results can provide hydrodynamic pressure and flow velocity boundary conditions for further research on the dynamic evolution process of water damage shape of ballastless track.

\section{Data Availability}

The data used to support the findings of this study are available from the corresponding author upon request.

\section{Conflicts of Interest}

The authors declare that there are no conflicts of interest regarding the publication of this paper.

\section{Acknowledgments}

We gratefully acknowledge the financial support provided by the Foundation for High-Level Talents of Henan University of Technology (2018BS024), the Program for Innovative Research Team of Science \& Technology of Henan Province of China (19IRTSTHN020), and the National Natural Science Foundation of China (no. 51778543, no. U1434208). 


\section{References}

[1] P.-E. Gautier, "Slab track: Review of existing systems and optimization potentials including very high speed," Construction and Building Materials, vol. 92, pp. 9-15, 2015.

[2] L. Tian, "Systematic design of ballastless track on passenger dedicated lines," Zhongguo Tiedao Kexue/China Railway Science, vol. 29, no. 1, pp. 13-16, 2008.

[3] D. Liu, Durability of ballastless slab track subjected to dynamic load, Southwest Jiaotong University, 2017.

[4] H. Wang, R. Chen, S. Qi, W. Cheng, and Y. Cui, "Long-Term Performance of Pile-Supported Ballastless Track-Bed at Various Water Levels," Journal of Geotechnical and Geoenvironmental Engineering, vol. 144, no. 6, p. 04018035, 2018.

[5] J. Ren, S. Deng, Z. Jin, J. Yang, and X. Liu, "Energy Method Solution for the Vertical Deformation of Longitudinally Coupled Prefabricated Slab Track," Mathematical Problems in Engineering, vol. 2017, pp. 1-11, 2017.

[6] C. Esveld, "Recent developments in slab track," European Railway Review, vol. 9, no. 2, pp. 81-85, 2003.

[7] Y. Peng, Analysis on the parameter influences and research on the improvement measures of track slab up warp of double-block ballastless track, Southwest Jiaotong University, 2013.

[8] Y. Harada, S. Tottori, N. Itai, and T. Noto, "Development of cement-asphalt mortar for slab tracks in cold climate," Railway Technical Research Institute, vol. 24, no. 2, pp. 62-67, 1983.

[9] J. Eisenmann and G. Leykauf, "Feste fahrbahn für schienenbahnen," in Betonkalender, vol. 2, pp. 291-326, 2000.

[10] DIN 1045-1 (07.2001), Concrete, reinforced and prestressed concrete structures.

[11] D. Bilow and D. Li, "Concrete slab track test on the high tonnage loop at the transportation technology center," in Proceedings of the AREMA Annual Conference '05, 2005.

[12] X. Cheng and J. Dong, "Analyses of the cause for the fracture of monolithic concrete bed in tunnel and treatment measures," Journal of Railway Engineering Society, vol. 128, no. 5, pp. 64-68, 2009.

[13] J. Ren, R. Xiang, and B. Lechner, "An innovative slab track testline in China. Bearing Capacity of Roads, Railways and Airfields," in 8th International Conference (BCR2A'09), University of Illinois, Urbana-Champaign, 2009.

[14] X.-P. Cai, W. Liu, P. Wang, and X. Ning, "Effect of land subsidence on regularity of double-block ballastless track," Gongcheng Lixue/Engineering Mechanics, vol. 31, no. 9, pp. 160165, 2014.

[15] Q. Xu, Y. Meng, B. Li, P. Lou, and B. Yan, "Fatigue stress spectrum of longitudinally connected ballastless track under temperature gradient," Zhongnan Daxue Xuebao (Ziran Kexue Ban)/Journal of Central South University (Science and Technology), vol. 46, no. 2, pp. 736-741, 2015.

[16] P. Lou, J. Zhu, G. Dai, and B. Yan, "Experimental study on bridge-track system temperature actions for Chinese highspeed railway," Archives of Civil and Mechanical Engineering, vol. 18, no. 2, pp. 451-464, 2018.

[17] G. Long, H. Liu, K. Ma, Y. Xie, and W. Li, "Development of High-Performance Self-Compacting Concrete Applied as the Filling Layer of High-Speed Railway," Journal of Materials in Civil Engineering, vol. 30, no. 2, p. 04017268, 2018.

[18] F. Javanmardi, P. Léger, and R. Tinawi, "Seismic structural stability of concrete gravity dams considering transient uplift pressures in cracks," Engineering Structures, vol. 27, no. 4, pp. 616-628, 2005.
[19] S. Cao, R. Yang, C. Su, F. Dai, X. Liu, and X. Jiang, "Damage mechanism of slab track under the coupling effects of train load and water," Engineering Fracture Mechanics, vol. 163, pp. 160175,2016

[20] R. Tinawi and L. Guizani, "Formulation of hydrodynamic pressures in cracks due to earthquakes in concrete dams," Earthquake Engineering \& Structural Dynamics, vol. 23, no. 7, pp. 699-715, 1994.

[21] J. Sun and Q. Yao, "Experiment and theory study on brittle damage constitutive model of ordinary strength high performance concrete," Jianzhu Jiegou Xuebao/Journal of Building Structures, vol. 30, no. 2, pp. 309-314, 2009.

[22] X. Liu, P. Zhao, R. Yang et al., Design theory and methods for ballastless track of passenger dedicated line, Southwest Jiaotong Press, 2010.

[23] H. Liu, Fluid Mechanics, China building industry press, 2004. 


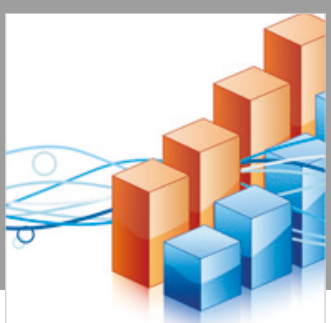

Advances in

Operations Research

\section{-n-m}
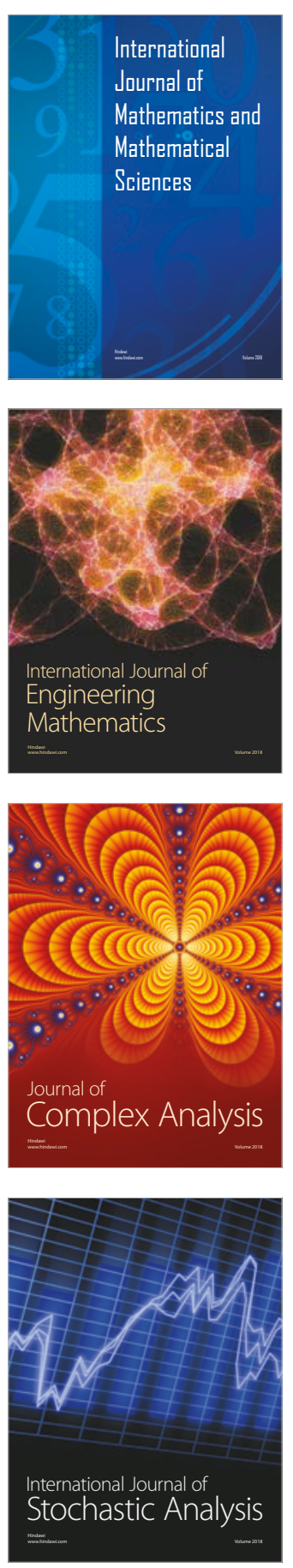
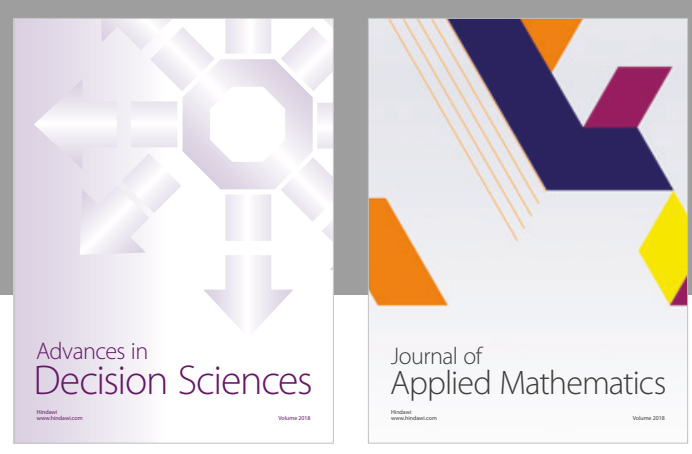

Journal of

Applied Mathematics
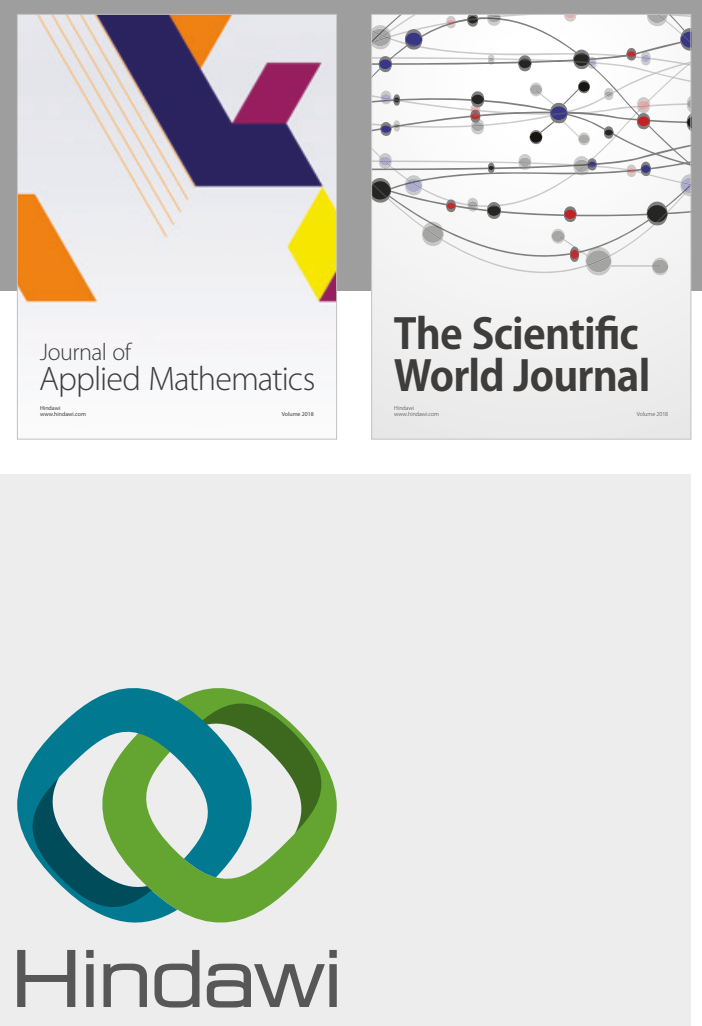

Submit your manuscripts at

www.hindawi.com

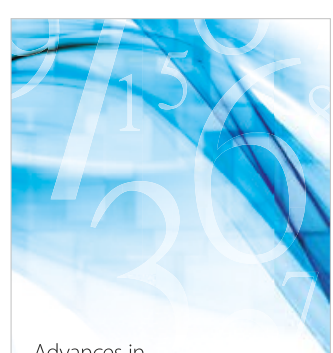

Advances in
Numerical Analysis
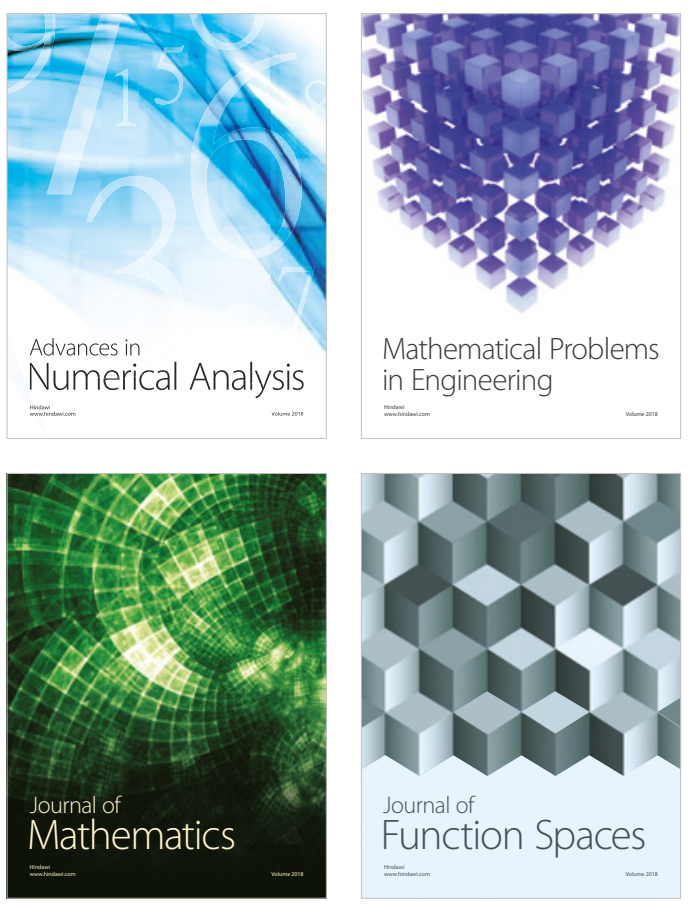

Mathematical Problems in Engineering

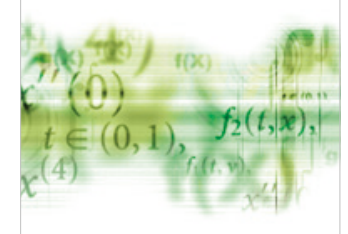

International Journal of

Differential Equations

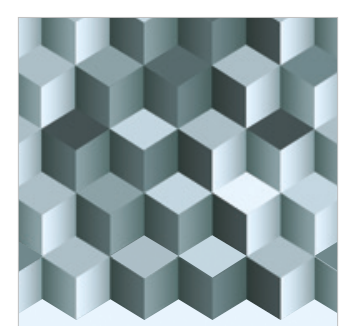

Journal of

Function Spaces

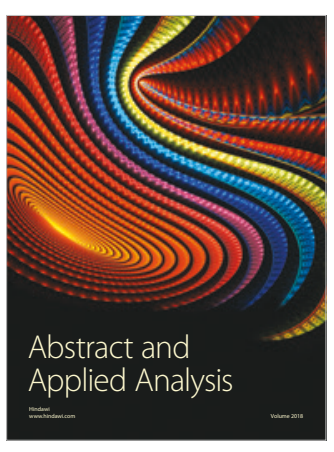

The Scientific

World Journal

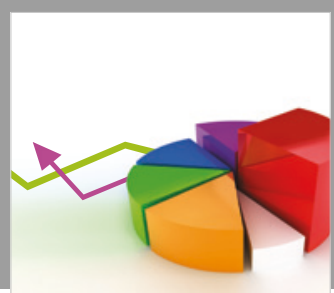

Journal of

Probability and Statistics
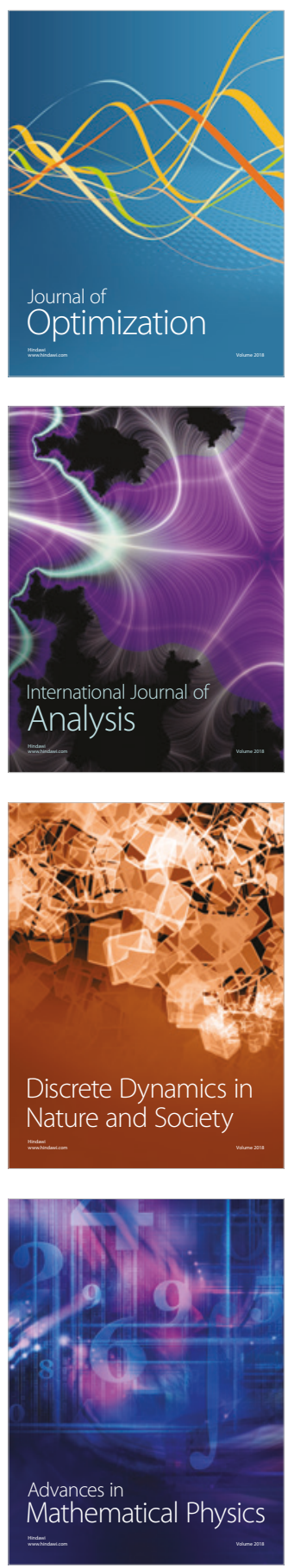